\title{
Intraoperative Indocyanine Green Video Angiography (ICG-VA) with FLOW 800 Software in Complex Intracranial Aneurysm Surgery
}

\section{Tao Xue}

The First Affiliated Hospital of Soochow University

\section{Ruming Deng}

Department of Neurosurgery, Bozhou People's Hospital

\section{Bixi Gao}

The First Affiliated Hospital of Soochow University

\section{Zilan Wang}

The First Affiliated Hospital of Soochow University

\section{Chao Ma}

The First Affiliated Hospital of Soochow University

\section{Wanchun You}

The First Affiliated Hospital of Soochow University

\section{Yun Zhu}

The First Affiliated Hospital of Soochow University

\section{Zhouqing Chen}

The First Affiliated Hospital of Soochow University

Zhong Wang ( $\nabla$ wangzhong761@163.com )

The First Affiliated Hospital of Soochow University

\section{Research}

Keywords: aneurysm, bypass, clipping, FLOW 800 ICG-VA

Posted Date: November 17th, 2020

DOl: https://doi.org/10.21203/rs.3.rs-104879/v1

License: (c) (i) This work is licensed under a Creative Commons Attribution 4.0 International License. Read Full License

Version of Record: A version of this preprint was published at Chinese Neurosurgical Journal on June 1st, 2021. See the published version at https://doi.org/10.1186/s41016-021-00247-z. 


\section{Abstract}

Objective: Indocyanine green video angiography (ICG-VA) is a safe and effective instrument to assess changes in cerebral blood flow during cerebrovascular surgery. After ICG-VA, FLOW 800 provides a colorcoded map to directly observe the dynamic distribution of blood flow and to calculate semiquantitative blood flow parameters later. The purpose of our study is to assess whether FLOW 800 is useful for surgery of complex intracranial aneurysms and to provide reliable evidence for intraoperative decision making.

Methods: We retrospectively reviewed patients with complex aneurysms that underwent microsurgical and intraoperative evaluation of ICG-VA and FLOW 800 color-coded maps from February 2019 to May 2020. FLOW 800 data were correlated with patient characteristics, clinical outcomes, and intraoperative decision-making.

Results: The study included 32 patients with 42 complex aneurysms. All patients underwent ICG-VA FLOW 800 data provided semiquantitative data regarding localization, flow status in major feeding arteries; color maps confirmed relative adequate flow in parent, branching and bypass vessels.

Conclusions: FLOW 800 is a useful supplement to ICG-VA for intraoperative cerebral blood flow assessment. ICG-VA and FLOW 800 can help to determine the blood flow status of the parent artery after aneurysm clipping and the bypass vessels after aneurysm bypass surgery.

\section{Introduction}

Complex cerebral aneurysms contain not only giant aneurysms (greater than $25 \mathrm{~mm}$ in diameter) but also some aneurysms characterized by large (15-25 mm), difficult locations, previous treatment, presence or absence of collateral circulation, intraluminal thrombus and calcification of the aneurysmal wall[ ${ }^{[1]}$. To discuss the selection of endovascular or surgical treatment for complex intracranial aneurysms is beyond the aim of this article. However, the treatment method should be decided carefully with the consideration of advanced endovascular technology such as modified stents and novel embolic agents perhaps improving therapeutic effect for these complex aneurysms ${ }^{[2]}$. Once the surgical procedure is adopted, direct clipping becomes the most common strategy ${ }^{[3]}$. When the direct approach is impractical or at-risk, alternative approaches including trapping with or without flow replacement (bypass), primary proximal vessel occlusion (Hunterian ligation) and distal vessel occlusion can be used ${ }^{[4]}$. The complexity of these aneurysms often makes the operation more difficult, and they are accompanied by a high occurrence of complications. In consequence, some intraoperative assistant equipment is required to avoid such lesions.

Indocyanine green video angiography (ICG-VA) has been widely used in vascular neurosurgery, especially in cases of aneurysms, arteriovenous malformations (AVMs) and bypass, to assess the cerebral blood flow since its introduction in $2003^{[5-9]}$. The potential disadvantages of conventional ICG-VA include that it 
only evaluates the blood flow within the field of view and lacks quantitative analysis of the angiography images $^{[10]}$.

FLOW 800 (Carl Zeiss, Oberkochen, Germany), a new surgical microscope-integrated software program, allows ICG-VA data to be analyzed in a semiquantitative way by creating a color-coded map ${ }^{[11]}$. The colorcoded map identifies the direction and sequence of blood flow in the surrounding vasculature based on fluorescence dynamics after ICG injection. Surgeons can comparatively evaluate the flow rate at userdefined regions of interest (ROIs) within the operative field by the analysis of fluorescence profiles ${ }^{[11]}$. The application of FLOW 800 for microsurgical treatment of AVMs, regular intracranial aneurysms and moyamoya disease (MMD) has been reported in some research ${ }^{[11-15]}$, and a case report including 3 patients of complex intracranial aneurysms can be obtained in the available literature ${ }^{[16]}$. However, large studies for complex intracranial aneurysms are rare. The purpose of this study was to present our singlecenter experience with the application of intraoperative ICG-VA and hemodynamic analysis with Flow 800 in complex intracranial aneurysms.

\section{Material And Methods}

This is a single-center, retrospective study of consecutive patients that underwent microsurgical operations of complex intracranial aneurysms and intraoperative assessment of ICG-VA and FLOW 800 color-coded map from February 2019 to May 2020. The exclusion criteria include: (a) patients who were treated via endovascular methods alone, (b) patients allergic to ICG, (c) pediatric patients, (d) patients who had surgical treatment where FLOW 800 technology was not used. Based on the institutional guidelines, the ethics committee approval was not required for our study.

\section{Procedures details}

Before surgery, the patients underwent a routine computed tomography angiography (CTA) or/and digital subtraction angiography (DSA) scan to confirm aneurysm size, location, and morphology. The aneurysm treatment decision was made through a collaboration of vascular neurosurgeons and neurointerventionalists. Patients were considered for bypass if standard microsurgical clipping was not feasible. Surgical approaches, clippings, and bypasses were all performed in the standard way by the senior author. For approaches, a pterional or lateral supraorbital craniotomy was for aneurysms of the internal carotid artery (ICA), posterior communicating artery (PcomA), the middle cerebral artery (MCA), and the basilar artery (BA). Anterior communicating artery (AcomA) aneurysms were exposed via pterional, lateral supraorbital, or a midline approach. The anterior inferior cerebellar artery (AICA) and posterior inferior cerebellar artery (PICA) aneurysms were clipped by a suboccipital approach. The posterior cerebral artery (PCA) and the superior cerebellar artery (SCA) aneurysms were clipped after subtemporal craniotomy. For clipping, we carefully dissected the arachnoid membrane to achieve the proximal control of the main arteries until a temporary clip was able to be placed. After the aneurysm neck and dome were exposed completely, the surgical findings were consistent with the preoperative angiography to consider a clipping strategy and to select appropriate aneurysm clips. For bypass, 
ICA/external carotid artery (ECA)-radial artery (RA)-second/third segment of the MCA (M2/M3) bypass, ECA-RA-PCA, or superficial temporal artery (STA)-M2/M3/M4 bypass were usually performed to compensate for the sacrificed blood flow when the aneurysm was isolated or to improve the existing symptoms of cerebral ischemia. Anastomoses were performed under anesthetic burst suppression and with the patient's systolic blood pressure 10 to $20 \mathrm{mmHg}$ higher than the baseline during temporary occlusion. For intraoperative assessment of aneurysm occlusion, parent, and bypass artery patency, ICGVA with/without microdoppler ultrasound was performed. After surgery, some patients were admitted to an intensive care unit on basis of individual situation. Routine cranial computed tomography (CT) scans were performed to exclude rebleeding immediately after recovery from general anesthesia and cerebral infarction on the first postoperative day. Transcranial doppler (TCD) ultrasound was performed daily in patients with SAH to monitor the blood flow velocity. Cerebral vasospasm occurred when a mean blood flow velocity $\geq 120 \mathrm{~cm} / \mathrm{s}$ and/or an augment by $\geq 50 \mathrm{~cm} / \mathrm{s}$ within 24 hours $^{[17]}$. For patients with possible cerebral vasospasm or other neurological deficits, an urgent cranial CT scan with angiography and perfusion was performed additionally.

\section{Fluorescence angiography and FLOW 800 analysis}

An OPMI Pentero surgical microscope with integrated ICG technology (Carl Zeiss GmbH, Oberkochen, Germany) was used for intraoperative analysis of cerebral blood flow. The application of ICG for microsurgery was proven technique and has been described previously in detail ${ }^{[18]}$. After $25 \mathrm{mg}$ of ICG was administered intravenously, fluorescence could be induced by the integrated light source (about $800 \mathrm{~mm}$ in wavelength) and filmed by integrated camera as INFRARED 800 (IR 800) video which enabled a real-time assessment of arterial, cortical, and venous blood flow under visual field. The times of ICG-VA runs was decided by neurosurgeon. In general, intraoperative ICG-VA was performed twice at baseline and after aneurysm treatment.

The FLOW 800 analysis software (Release 2.21, Carl Zeiss GmbH, Oberkochen, Germany) was also integrated with the surgical microscope, allowing for real-time and semi-quantitative analysis of the cerebral blood flow via IR 800 video. After injection of ICG, the software steadily records the relative fluorescence intensity during the whole process of ICG-VA and automatically converts the IR 800 video into a color-coded map, which provides an overview on the sequence when fluorescence appears at what part of the video image. An intuitive scale highlights early appearance of fluorescence in red (e.g. arteries), medium appearance in yellow/green (e.g. cortical capillaries) and late appearance in blue (e.g. veins). The color-coded maps generated by FLOW 800 software simply provide clearer indications rather than absolute measurements. To receive detailed fluorescence intensity curve of a specific vessel or area within the field of view, up to 8 definable regions of interest (ROIs) can be selected and analyzed. In our study, ICG-VA and FLOW 800 analysis were performed as clinical indications. The fluorescence curves

which have been thoroughly studied by previous studies ${ }^{[14-16]}$ were not analyzed because these analyses take longer, which is not available or practical during surgery. In other word, the fluorescence curves of ROls can provide hemodynamic analysis of the vessels but we did not use them during our operation and depended only on the FLOW 800 color-coded map that is effective in guiding intraoperative procedures. 


\section{Data collection}

The following variables of every patient were collected retrospectively: age, gender, subarachnoid hemorrhage (SAH), Glasgow Coma Scale (GCS), Hunt-Hess grade, modified Rankin Score (mRS), aneurysm number, size, complexity, location, intraoperative findings, FLOW 800 results, postoperative imaging, and complications.

\section{Results}

\section{Patient and aneurysm characteristics}

A total of 32 consecutive patients with 42 complex aneurysms were enrolled in this study. Patients ranged in age from 17 to 77 years (mean age of 54 years) with a gender ratio of 1:5.4 (male/female). Thereof, 16 patients were treated for unruptured aneurysms and 16 patients with SAH were treated for a ruptured aneurysm. Baseline patient characteristics including GCS, Hunt-Hess grade, and mRS are summarized in Table 1. 
Table 1

Baseline patient and aneurysm characteristics

\section{Variable}

Age (years)

Mean \pm SD

Range

Gender (\%)

Female

Male

SAH (\%)

GCS (\%)

$0-3$

4-6

7-9

$10-12$

$13-15$

Hunt-Hess grade (\%)

0 (unruptured)

mRS (\%)
1

2

3

4

5

2

3

5
Value $(n=32)$

$53.91 \pm 14.45$

17-77

27 (84\%)

$5(16 \%)$

16 (50\%)

0 (0\%)

$2(6 \%)$

2 (6\%)

3 (9\%)

25 (78\%)
16 (50\%)

$5(16 \%)$

$4(12 \%)$

$5(16 \%)$

2 (6\%)

$0(0 \%)$

$\mathrm{SD}=$ standard deviation; $\mathrm{SAH}$ = subarachnoid hemorrhage; $\mathrm{GCS}=$ Glasgow Coma Scale; $\mathrm{mRS}=$ modified Rankin score; AVM = arteriovenous malformation; AComA = anterior communicating artery; $\mathrm{MCA}=$ middle cerebral artery; ICA = internal carotid artery; PComA = posterior communicating artery; $\mathrm{PICA}=$ posterior inferior cerebellar artery; $\mathrm{AICA}=$ anterior inferior cerebellar artery; $\mathrm{PCA}=$ posterior cerebral artery; SCA = superior cerebellar artery; BA = basilar artery. 


\begin{tabular}{|c|c|}
\hline Variable & Value $(n=32)$ \\
\hline 0 & $0(0 \%)$ \\
\hline 1 & $11(34 \%)$ \\
\hline 2 & $8(25 \%)$ \\
\hline 3 & $3(9 \%)$ \\
\hline 4 & $3(9 \%)$ \\
\hline 5 & $7(22 \%)$ \\
\hline Aneurysm number & 42 \\
\hline \multicolumn{2}{|l|}{ Aneurysm size (mm) } \\
\hline Mean \pm SD & $16.81 \pm 16.25$ \\
\hline Range & $1.6-74.7$ \\
\hline \multicolumn{2}{|l|}{ Complexity (one aneurysm may have several complexity) } \\
\hline Giant ( $\geq 25 \mathrm{~mm})$ & 6 \\
\hline Large (15 mm-25 mm) & 6 \\
\hline Difficult locations & 14 \\
\hline Presence of collateral circulation & 4 \\
\hline Intraluminal thrombus & 3 \\
\hline Calcification of wall & 3 \\
\hline Previous treatment & 2 \\
\hline Others & 8 \\
\hline Multiple aneurysms requiring simultaneous management & 5 (patients) \\
\hline With sellar tumor & 1 \\
\hline Wide neck & 1 \\
\hline Dissecting aneurysm & 1 \\
\hline
\end{tabular}

$\mathrm{SD}=$ standard deviation; $\mathrm{SAH}$ = subarachnoid hemorrhage; $\mathrm{GCS}=$ Glasgow Coma Scale; $\mathrm{mRS}$ = modified Rankin score; AVM = arteriovenous malformation; $\mathrm{AComA}$ = anterior communicating artery; $\mathrm{MCA}=$ middle cerebral artery; ICA = internal carotid artery; PComA = posterior communicating artery; $\mathrm{PICA}=$ posterior inferior cerebellar artery; $\mathrm{AICA}=$ anterior inferior cerebellar artery; $\mathrm{PCA}=$ posterior cerebral artery; SCA = superior cerebellar artery; BA = basilar artery. 


\begin{tabular}{|c|c|}
\hline Variable & Value $(n=32)$ \\
\hline Anterior circulation & $32(76 \%)$ \\
\hline AComA & $3(7 \%)$ \\
\hline MCA & $9(21 \%)$ \\
\hline ICA-PComA & 7 (17\%) \\
\hline ICA & $13(31 \%)$ \\
\hline Posterior circulation & $10(24 \%)$ \\
\hline PICA & $1(2 \%)$ \\
\hline AICA & $2(5 \%)$ \\
\hline PCA & $2(5 \%)$ \\
\hline SCA & $1(2 \%)$ \\
\hline BA & $4(10 \%)$ \\
\hline \multicolumn{2}{|c|}{$\begin{array}{l}\text { SD = standard deviation; } \mathrm{SAH}=\text { subarachnoid hemorrhage; GCS = Glasgow Coma Scale; } \mathrm{mRS}= \\
\text { modified Rankin score; AVM = arteriovenous malformation; } \mathrm{AComA}=\text { anterior communicating arter } \\
\text { MCA = middle cerebral artery; ICA = internal carotid artery; PComA = posterior communicating artery } \\
\text { PICA = posterior inferior cerebellar artery; AICA = anterior inferior cerebellar artery; PCA = posterior } \\
\text { cerebral artery; SCA = superior cerebellar artery; BA = basilar artery. }\end{array}$} \\
\hline
\end{tabular}

The mean aneurysm size was $16.81 \pm 16.25 \mathrm{~mm}$, ranged from 1.6 to $74.7 \mathrm{~mm}$. Of 42 aneurysms, 32 (76\%) aneurysms were located at the anterior circulation including $3(7 \%)$ at the anterior communicating artery, $9(21 \%)$ at the middle cerebral artery, $7(17 \%)$ at the posterior communicating artery and $13(31 \%)$ at the internal carotid artery; $10(24 \%)$ aneurysms were located at the posterior circulation comprised of 1 $(2 \%)$ at the posterior inferior cerebellar artery, $2(5 \%)$ at the anterior inferior cerebellar artery, $2(5 \%)$ at the posterior cerebral artery, $1(2 \%)$ at the superior cerebellar artery and $4(10 \%)$ at the basilar artery. Baseline aneurysm characteristics including the complexity of included aneurysms are listed in Table 1.

\section{Representative cases}

We presented the following three cases to illustrate the values of ICG-VA with Flow 800 analysis in complex intracranial aneurysm surgery. In addition, the details of the operative information and FLOW 800 findings for individual cases were summarized in Table 2 . 
Table 2

Details of FLOW 800 findings for all cases with associated operative information

No. Pathology Operation

Complexity of aneurysms, intraoperative strategy, and Flow 800 findings paraclinoid

aneurysm
$1 \quad$ Left ICA C5

Clipping

The aneurysm was located under the anterior clinoid and we grinded the bone of the anterior clinoid carefully to expose the aneurysm. A cross-window clip was applied because the aneurysm points to the inside. FLOW 800 analysis confirmed complete occlusion of aneurysm and adequate flow in parent artery.

2 Left ICA C6 Trapping + high

large flow EC-IC bypass

recurrent

aneurysm

The aneurysm was $16.6 * 12.3 \mathrm{~mm}$ in size and had previous treatment. We trapped the aneurysm at left ICA initial part and ICA before PComA branch after high flow EC-IC bypass (M3-RA-superior thyroid artery). FLOW 800 analysis indicated the bypass vascular was fluent and peripheral blood flow improved.

The aneurysm was $25 * 20 \mathrm{~mm}$ in size and the patient also had left CCF. We performed high flow EC-IC bypass (M2RA-ICA). Then, we trapped aneurysm at ICA initial part and ICA before PComA branching. FLOW 800 analysis and intraoperative ultrasound showed the aneurysm disappeared and the bypass vascular was fluent.

The aneurysm was ruptured with much bleeding and $25 * 15 \mathrm{~mm}$ in size. We first trapped aneurysm at proximal MCA and distal MCA to control bleeding. Then we excised the aneurysm and performed high flow EC-IC bypass (M2RA-ECA). FLOW 800 analysis and intraoperative ultrasound confirmed the bypass vascular was fluent. Afterwards, distal MCA was released and FLOW 800 was used again to confirm the bypass vascular was unobstructed.

The aneurysm was $40 * 22 \mathrm{~mm}$ in size. high flow EC-IC bypass (M3-RA-superior thyroid artery) was applied to this patient before the aneurysm was trapped. FLOW 800 analysis exhibited the bypass vessel was fluent.

The aneurysm was about $24 \mathrm{~mm}$ in diameter. Combination of two clips $(762,782)$ was used to mold and clip the giant aneurysm. The first FLOW 800 detected the aneurysm was clipped completely but the parent artery was stenosis. Then, we repositioned the second clip and FLOW 800 and intraoperative TCD both indicated the blood flow of parent artery recovered.

ICA = internal carotid artery; high flow EC-IC = extracranial-to-intracranial; PComA = posterior communicating artery; AComA = anterior communicating artery; CCF = carotid-cavernous fistula; MCA = middle cerebral artery; ECA = external carotid artery; ICG-VA = Indocyanine green video angiography; $\mathrm{VA}$ = vertebral artery; PICA = posterior inferior cerebellar artery; $\mathrm{AVM}=$ arteriovenous malformation; $\mathrm{ACA}=$ anterior cerebral artery; $\mathrm{AChA}=$ anterior choroidal artery; $\mathrm{BA}=$ basilar artery; $\mathrm{LSO}=$ lateral supraorbital; AICA = anterior inferior cerebellar artery; STA = superficial temporal artery; SCA = superior cerebellar artery; PCA = posterior cerebral artery; TCD = transcranial doppler. 
No. Pathology Operation

7 AcomA Aneurysmectomy aneurysm

$\begin{array}{ll}\text { Left VA V4- } & \text { Clipping + high } \\ \text { BA giant } & \text { flow EC-IC bypass } \\ \text { dissecting } & \\ \text { + left PCA } & \\ \text { P2 } & \\ \text { fusiform + } & \\ \text { left PComA } & \\ \text { + bilateral } & \\ \text { ICA C1 } & \\ \text { multiple } & \\ \text { aneurysms } & \end{array}$

\section{Complexity of aneurysms, intraoperative strategy, and Flow 800 findings}

The aneurysm $\left(23^{*} 15 \mathrm{~mm}\right)$ had intraluminal thrombus and calcification of the aneurysmal wall. First, we separated the aneurysm neck and ipsilateral A2, temporary blocked ipsilateral A1, A2, positioned an aneurysm clip, loosened the temporary block, FLOW 800 indicated A2 without blood flow, debugged aneurysm clip, A2 still had no blood flow. Then, ipsilateral A1 and A2 were blocked again, the aneurysm was cut open, intraaneurysm thrombus was removed, and aneurysm was resected. Contralateral $\mathrm{A} 1$, anterior communicating artery and A2 were seen below. The aneurysm neck was thin and was not able to be clipped. Therefore, we tried to suture anterior traffic artery and ipsilateral A2 blood vessel. After suture, release temporary block, blood flow was acceptable. However, oozing blood was found, and suture was added. We removed residual aneurysm, detected ipsilateral A2 again and found no blood flow signal by FLOW 800. Considering intravascular thrombosis, we dismantled anastomosis, removed the fresh blood clots, used heparin saline flushing repeatedly, saw the endangium injury and sutured blood vessels again. Afterwards, we opened suture again, still saw a thrombosis, loosened the A2 temporary block, saw the retrograde bleeding obvious, considered the remote vessels well compensated, and hence gave up blood vessel suture, Finally, we closed ipsilateral A1, A2 and AcomA.

We clipped the left PComA aneurysm with a 644 crosswindow right-angle clip. FLOW 800 analysis showed the parent artery was fluent. Then, we performed high flow EC-IC bypass (PCA-RA-superior thyroid artery) and FLOW 800 analysis indicated the bypass vessel was unobstructed.

The patient's condition was extremely complex. Multiple aneurysms were not ruptured, but the vertebrobasilar dissection aneurysm was huge with intraluminal thrombosis. The patient also had obvious neurological defect like posterior circulation ischemia and cranial nerve damage. One operation was difficult to deal with multiple intracranial aneurysms. After discussion in the department, the left ECA-RA-PCA bypass plus left PComA aneurysm clipping were performed, and the left VA V4-BA giant dissecting aneurysm was occluded by intravascular balloon. Left PCA aneurysm was difficult to deal with because its shape (fusiform) and bilateral ICA C1 aneurysms were not handled at this time.

$\mathrm{ICA}=$ internal carotid artery; high flow $\mathrm{EC}-\mathrm{IC}=$ extracranial-to-intracranial; PComA = posterior communicating artery; $\mathrm{AComA}$ = anterior communicating artery; $\mathrm{CCF}$ = carotid-cavernous fistula; $\mathrm{MCA}$ = middle cerebral artery; ECA = external carotid artery; ICG-VA = Indocyanine green video angiography; $\mathrm{VA}$ = vertebral artery; PICA = posterior inferior cerebellar artery; AVM = arteriovenous malformation; $\mathrm{ACA}=$ anterior cerebral artery; $\mathrm{AChA}$ = anterior choroidal artery; BA = basilar artery; $\mathrm{LSO}=$ lateral supraorbital; AICA = anterior inferior cerebellar artery; STA = superficial temporal artery; SCA = superior cerebellar artery; PCA = posterior cerebral artery; TCD = transcranial doppler. 
No. Pathology Operation

Complexity of aneurysms, intraoperative strategy, and Flow 800 findings

9

$\begin{array}{ll}\text { Right } & \text { Clipping } \\ \text { ruptured } & \\ \text { MCA + right } & \\ \text { AcomA + } & \\ \text { left ICA C6 } & \\ \text { multiple } & \\ \text { aneurysms } & \end{array}$

MCA + right

AcomA +

left ICA C6

aneurysms
Right Clipping

\begin{abstract}
We prepared to deal with the right ruptured MCA aneurysm and right AcomA aneurysm through right pterional approach. Firstly, the right MCA aneurysm was clipped satisfactorily with a 940-aneurysm clip and the branches of the MCA were satisfactorily preserved, which were confirmed by FLOW 800 analysis. Then, the A1 segments of the bilateral ACAs were exposed and temporarily blocked. We separated the right ACA aneurysm neck and positioned a 742-aneurysm clip. However, it was found that the clipping was not complete, and the 742-aneurysm clip was replaced by a $940-$ aneurysm clip. The residual in the aneurysm neck was strengthened with two mini clips. Flow 800 indicated the aneurysm was clipped completely, and the bilateral A1 and $A 2$ vessels were unobstructed.
\end{abstract}

10
Right PICA aneurysm
Clipping The aneurysm was exposed after we separated along the
proximal to the distal end of right PICA. Then, the aneurysm was clipped and the parent artery was preserved, which were verified by Flow 800 analysis.

\section{1}

$\begin{array}{ll}\text { Right } & \text { Clipping } \\ \text { PcomA }+ & \\ \text { AChA } & \\ \text { multiple } & \\ \text { aneurysms } & \end{array}$

12 BA apex
aneurysm

\begin{abstract}
A saccular aneurysm could be seen at about $5 \mathrm{~mm}$ from the bifurcation of right ICA, pointing to the outside, and the AChA went out at the proximal of the aneurysm. Further exploration to the proximal of ICA showed that there was a lobulated aneurysm at the dorsal side of right PcomA, pointing to the posterior inferior and posterior lateral side respectively. The, we separated the PcomA and its surrounding branches. The PcomA aneurysm was clipped by a transvascular T-Bar-shaped aneurysm clip satisfactorily, and then the AChA aneurysm was clipped satisfactorily by a 940 -aneurysm clip. Flow 800 analysis revealed that no parent artery stenosis was found in both places, and the branch vessels were well preserved.
\end{abstract}

The right lateral supraorbital (LSO) approach was used to open the second space and the superior space of the internal carotid artery. Then we cut the liliequis membrane, exposed the posterior clinoid process, and showed the BA apex aneurysm, pointing upward, about the size of $4 \mathrm{~mm}$. After that we separated the aneurysm neck and clipped the aneurysm with one 940 -aneurysm clip (Aesculap, Germany) satisfactorily. Finally, FLOW 800 analysis indicated complete occlusion of the aneurysm and sufficient flow in parent artery.

$\mathrm{ICA}=$ internal carotid artery; high flow EC-IC = extracranial-to-intracranial; PComA = posterior communicating artery; $\mathrm{AComA}=$ anterior communicating artery; $\mathrm{CCF}=$ carotid-cavernous fistula; $\mathrm{MCA}$ = middle cerebral artery; ECA = external carotid artery; ICG-VA = Indocyanine green video angiography; $\mathrm{VA}$ = vertebral artery; PICA = posterior inferior cerebellar artery; $\mathrm{AVM}=$ arteriovenous malformation; $\mathrm{ACA}=$ anterior cerebral artery; $\mathrm{AChA}=$ anterior choroidal artery; $\mathrm{BA}=$ basilar artery; $\mathrm{LSO}=$ lateral supraorbital; AICA = anterior inferior cerebellar artery; STA = superficial temporal artery; SCA = superior cerebellar artery; PCA = posterior cerebral artery; TCD = transcranial doppler. 
No. Pathology Operation

13

AlCA distal

ruptured

aneurysm

Clipping +

trapping +

aneurysmectomy

\section{Complexity of aneurysms, intraoperative strategy, and Flow 800 findings}

There was a small amount of old hematoma on the left lateral side of the medulla. After removal, the aneurysm with high tension, adhesion with the posterior cranial nerve, was carefully separated. The parent artery was thin. The aneurysm ruptured again during separation and the parent artery was blocked. Eventually, the aneurysm was trapped and resected. FLOW 800 analysis demonstrated the aneurysm disappeared.

14 Left PcomA Clipping aneurysm

with intraluminal thrombus

During the separation, the aneurysm ruptured and the proximal ICA was temporarily blocked. We clipped the aneurysm with 752 (Aesculap, Germany) aneurysm clip. Intraluminal thrombus was seen during the operation. After adjusting the aneurysm clip slightly, the ICA, PComA and AChA were examined for distortion. FLOW 800 analysis was performed to confirm the blood flow was unobstructed, and no residual of aneurysm was found.

Firstly, the end-to-side anastomosis between the parietal branch of STA and M4 segment of MCA was performed under microscope. TCD monitoring showed that the anastomosed vessels were unobstructed and the pulse was good. Then, we separated the lateral fissure and the arachnoid of the skull base, lifted the temporal lobe, and found the giant aneurysm (54*45 $\mathrm{mm}$ in size). ICA in the neck and distal end of the parent artery were temporarily blocked. Afterwards, the aneurysm was cut open, thrombus and organized tissue were removed, and most of the aneurysm was resected. Two aneurysm clips were positioned along the aneurysm neck and then we loosened the temporary blocking. The intraoperative electrophysiological monitoring showed nothing abnormal was detected. Intraoperative TCD and FLOW 800 analysis confirmed that the blood flow of the parent artery and bypass vessel was unobstructed. The giant aneurysm disappeared.

When the second space was opened, trunk of the basilar artery could be seen on the deep side. After further separation and enlargement of the space, it could be seen that the aneurysm was located at the intersection of the BA and the right SCA. Then, we separated the aneurysm neck carefully, and clipped the aneurysm satisfactorily by a 940 clip. There was no stenosis in the BA and SCA. FLOW 800 analysis indicated that the aneurysm was completely occlusive and the blood flow of BA, SCA and PCA was fluent.

ICA = internal carotid artery; high flow EC-IC = extracranial-to-intracranial; PComA = posterior communicating artery; AComA = anterior communicating artery; CCF = carotid-cavernous fistula; MCA = middle cerebral artery; ECA = external carotid artery; ICG-VA = Indocyanine green video angiography; $\mathrm{VA}$ = vertebral artery; PICA = posterior inferior cerebellar artery; $\mathrm{AVM}=$ arteriovenous malformation; $\mathrm{ACA}=$ anterior cerebral artery; $\mathrm{AChA}=$ anterior choroidal artery; $\mathrm{BA}=$ basilar artery; $\mathrm{LSO}=$ lateral supraorbital; AICA = anterior inferior cerebellar artery; STA = superficial temporal artery; SCA = superior cerebellar artery; PCA = posterior cerebral artery; TCD = transcranial doppler. 
No. Pathology Operation

17

Right MCA

multiple

large

aneurysms

Clipping + aneurysmectomy + trapping + low flow EC-IC bypass
Complexity of aneurysms, intraoperative strategy, and Flow 800 findings
Two aneurysms were $18.10 * 14.74 \mathrm{~mm}$ and $18.02 * 13.87 \mathrm{~mm}$ in size respectively. After the M1 segment of right MCA was separated, one of the aneurysms was seen, which originated from the bifurcation of the temporal polar artery. Careful separation of the aneurysm showed that there was hyperplasia and calcification of the aneurysm wall. Then, we temporarily blocked the beginning of $\mathrm{M} 1$, and found obvious thickening of the aneurysm wall after incision of the aneurysm. After removing most of the aneurysm, we tried to clip the aneurysm with the combination of rightangle clips, cross-vessel clips, and other kinds of aneurysm clips. FLOW 800 analysis found that the blood flow signal in the parent artery was weak and repeated adjustment of the aneurysm clips failed to maintain the patency of the parent artery. The other aneurysm was located at the beginning of the temporal pole artery, and the temporal polar artery was located on the aneurysm. The aneurysm was trapped directly. Afterwards, low flow EC-IC bypass (STA-M4) was performed by end-to-side anastomosis. Intraoperative FLOW 800 analysis confirmed that the anastomosis was unobstructed.

There was a cystic lesion in the first space. The tumor capsule wall was cut after electrocoagulation and there was some yellowish liquid in it. The whole cyst wall was removed, and the normal pituitary gland and pituitary stalk were well protected. Then, we gently lifted the frontal lobe, saw the ICA, optic nerve, and carefully separated the arachnoid around ICA. The aneurysm was located under the anterior clinoid process. We carefully grinded the anterior clinoid to and exposed the proximal neck of the aneurysm. Afterwards, the aneurysm neck was separated and clipped with 640T, 854T and 644T (Aesculap, Germany) aneurysm clips. FLOW 800 exhibited that the aneurysm was clipped satisfactorily and there was no stenosis of the parent artery.

ICA = internal carotid artery; high flow EC-IC = extracranial-to-intracranial; PComA = posterior communicating artery; $\mathrm{AComA}$ = anterior communicating artery; $\mathrm{CCF}$ = carotid-cavernous fistula; $\mathrm{MCA}$ = middle cerebral artery; ECA = external carotid artery; ICG-VA = Indocyanine green video angiography; $\mathrm{VA}=$ vertebral artery; PICA = posterior inferior cerebellar artery; $\mathrm{AVM}=$ arteriovenous malformation; $\mathrm{ACA}=$ anterior cerebral artery; $\mathrm{AChA}$ = anterior choroidal artery; BA = basilar artery; $\mathrm{LSO}=$ lateral supraorbital; AICA = anterior inferior cerebellar artery; STA = superficial temporal artery; SCA = superior cerebellar artery; PCA = posterior cerebral artery; $\mathrm{TCD}=$ transcranial doppler. 
No. Pathology Operation

19

Left ICA C6 Clipping + low

large

aneurysm
Complexity of aneurysms, intraoperative strategy, and Flow 800 findings
At first, the aneurysm $\left(22.5^{\star} 20 \mathrm{~mm}\right.$ in size) was electrocoagulated and shrunk slightly. Then, it was clipped with two cross-window right-angle aneurysm clips (654), and there was still a small amount of bleeding. Afterwards, a 786 great curved aneurysm clips was used to strengthen the aneurysm. Intraoperative TCD monitoring and FLOW 800 analysis showed that the aneurysm was clipped completely and the blood flow was unobstructed by ICA, MCA, ACA. Finally, low flow EC-IC bypass (STA-M4) was performed and the second time FLOW 800 analysis confirmed that the bypass vessel was fluent.
20 Left PCA Clipping

ruptured aneurysm, left PComA aneurysm?
The initial part of the PComA was cystic dilated, and PComA was slender, starting from the wall of the cystic dilated vessel. There was adherent organized hematoma under the optic chiasma. After partial removal of the hematoma, it could be seen that one aneurysm was located at the beginning of the left PCA. The aneurysm neck was carefully separated and the aneurysm neck was exposed satisfactorily. A 742-aneurysm clip was used, and there was a small amount of bleeding in the process of clipping. A small amount of residual aneurysm was found in the distal end of the aneurysm. A 940 clip was applied. FLOW 800 analysis showed satisfactory clipping of the aneurysm and no stenosis of the parent artery. After consideration, the initial part of the PComA was dilated obviously, and the arterial wall was weak under the microscope. A mini clip was used to clamp the enlarged protuberant vessels.

We grinded the bone of the anterior clinoid carefully to expose the aneurysm. A cross-window clip was used to clamp the aneurysm. FLOW 800 analysis confirmed the blood flow of parent artery was unobstructed.

22 Left PComA Clipping aneurysm
The aneurysm was located outside of the left ICA, pointing outward and downward, with obvious peripheral adhesion. The PComA went out from the proximal aneurysm neck. We dissociated the aneurysm neck and clipped the aneurysm neck with a cross-window aneurysm clip. Intraoperative FLOW 800 analysis indicated that the clipping was satisfactory and the parent artery was unobstructed.

ICA = internal carotid artery; high flow EC-IC = extracranial-to-intracranial; PComA = posterior communicating artery; $\mathrm{AComA}$ = anterior communicating artery; $\mathrm{CCF}$ = carotid-cavernous fistula; $\mathrm{MCA}$ = middle cerebral artery; ECA = external carotid artery; ICG-VA = Indocyanine green video angiography; $\mathrm{VA}$ = vertebral artery; PICA = posterior inferior cerebellar artery; AVM = arteriovenous malformation; $\mathrm{ACA}$ = anterior cerebral artery; $\mathrm{AChA}=$ anterior choroidal artery; $\mathrm{BA}=$ basilar artery; $\mathrm{LSO}=$ lateral supraorbital; AICA = anterior inferior cerebellar artery; STA = superficial temporal artery; SCA = superior cerebellar artery; PCA = posterior cerebral artery; $\mathrm{TCD}$ = transcranial doppler. 
No. Pathology Operation

Complexity of aneurysms, intraoperative strategy, and Flow 800 findings

23

BA apex $\quad$ Clipping
and right
MCA M2
multiple
aneurysms

24

Left PComA Clipping aneurysm

with calcification

of wall

First, the BA apex aneurysm was clipped by a 752aneurysm clip and FLOW 800 analysis showed the aneurysm was clipped satisfactorily and bilateral PCAs, SCAs and other surrounding vessels were fluent. Then, a mini clip was applied to deal with the M2 aneurysm and FLOW 800 analysis showed the aneurysm was also clipped satisfactorily and the parent artery was unobstructed.

We took the incision of the left LSO approach. The aneurysm was located at the anterior skull base, and it was difficult to expose the aneurysm. We carefully detected that the aneurysm neck was located outside of the anterior clinoid process, and the aneurysm wall was calcified. After temporary blocking of ICA, the aneurysm was clipped with a 960-aneurysm clip, and then a straight aneurysm clip was given to strengthen the aneurysm. The aneurysm clipping was satisfactory. TCD and FLOW 800 analysis confirmed that the parent artery was unobstructed during the operation.

25 AComA Clipping ruptured aneurysm with previous treatment

Right MCA M2 bifurcation large aneurysm
Aneurysmectomy + trapping + low flow EC-IC bypass
The patient underwent clipping of AComA aneurysm 3 months ago. After exposing the ipsilateral A1, A2 and contralateral A1, A2, it was found that the original aneurysm was clipped well, and another AComA aneurysm ruptured. A straight aneurysm clip was used to clamp the aneurysm, and the aneurysm was satisfactorily clipped. No obvious vascular distortion was found in FLOW 800 analysis, and the blood flow of the ipsilateral A1, A2 and contralateral A1, A2 was unobstructed, and the aneurysm was not developed.

The aneurysm was about $20 * 18 \mathrm{~mm}$ in size, and two M3 branches emanated from the aneurysm. It was very difficult to shape and clip the aneurysm. Therefore, low flow EC-IC bypass (STA-M3) was performed and the blood flow was unobstructed confirmed by FLOW 800 . The aneurysm was isolated and resected. Finally, another intraoperative FLOW 800 indicated that M3 had blood flow, and bypass vessel was unobstructed.

ICA = internal carotid artery; high flow EC-IC = extracranial-to-intracranial; PComA = posterior communicating artery; $\mathrm{AComA}$ = anterior communicating artery; $\mathrm{CCF}=$ carotid-cavernous fistula; $\mathrm{MCA}$ = middle cerebral artery; ECA = external carotid artery; ICG-VA = Indocyanine green video angiography; $\mathrm{VA}=$ vertebral artery; PICA = posterior inferior cerebellar artery; $\mathrm{AVM}=$ arteriovenous malformation; $\mathrm{ACA}=$ anterior cerebral artery; $\mathrm{AChA}$ = anterior choroidal artery; BA = basilar artery; $\mathrm{LSO}=$ lateral supraorbital; AICA = anterior inferior cerebellar artery; STA = superficial temporal artery; SCA = superior cerebellar artery; PCA = posterior cerebral artery; $\mathrm{TCD}=$ transcranial doppler. 
No. Pathology Operation

\section{7}

Left MCA
bifurcation

ruptured

broad-base

aneurysm
Clipping + low

flow EC-IC bypass

\section{Complexity of aneurysms, intraoperative strategy, and Flow 800 findings}

The aneurysm $(10.7 * 9.8 \mathrm{~mm})$ was seen at the bifurcation of M1 segment of MCA, and the tension of aneurysm was high. We separated aneurysm with two branches M2 and found the aneurysm neck was wide. One M2 segment was involved, and the wall was thin, so conventional clipping was very difficult. We first performed low flow ECIC bypass (STA-M2). TCD monitoring showed that the anastomotic vessels were unobstructed and the pulse was good. Then, the aneurysm was reshaped to ensure the blood supply of another M2. Seven aneurysm clips $(950 T * 4,710 T * 2,960 T * 1)$ were used to reshape the aneurysm neck. Under the monitoring of TCD during operation, the positions of aneurysm clips were adjusted repeatedly to ensure that the blood flow of M1 segment and M2 segment was smooth, and FLOW 800 analysis was conducted to confirm the patency of blood flow. However, the blood flow of bypass vessel (STA-M2) was not detected again, and FLOW 800 analysis also indicated that the blood flow was blocked. Considering the anastomotic thrombosis, the anastomosis was opened, the fresh thrombus was removed, and the vessels were washed with heparin saline repeatedly. The vascular intima was intact, so the end-to-side anastomosis was performed between the frontal branch of STA and the original anastomosis. Finally, intraoperative FLOW 800 analysis and TCD exhibited the blood flow was unobstructed.

28 Right MCA M3 large aneurysm
Aneurysmectomy + trapping + low flow EC-IC bypass

The aneurysm ( $15^{\star} 12 \mathrm{~mm}$ in size) was in the $\mathrm{M} 3$ segment of the right MCA. We blocked M2 and M3 segments and resected the aneurysm. The STA was sutured end-to-end to the MCA-M3. Intraoperative TCD and FLOW 800 showed that the anastomotic vessels were unobstructed and the pulse was good.

29

$\begin{array}{ll}\text { Right } & \text { Clipping } \\ \text { ruptured } & \\ \text { MCA } & \\ \text { bifurcation } & \\ \text { large } & \\ \text { aneurysm } & \end{array}$

The aneurysm ( $20 * 15 \mathrm{~mm}$ in size) was in the M1 bifurcation of the right MCA. At first, the neck of the aneurysm was clipped with a 950-aneurysm clip, but the neck was too wide to be completely clipped. Then, a 960aneurysm clip was added to the lateral side, but it was still not completely clipped. We added another 742 and 786 aneurysm clips, adjusted clips repeatedly, and clipped the aneurysms. FLOW 800 analysis indicated clipping of the aneurysm was satisfactory, the blood flow of M1, M2 was unobstructed, and no obvious distortion of blood vessels was detected.

$\mathrm{ICA}=$ internal carotid artery; high flow $\mathrm{EC}-\mathrm{IC}=$ extracranial-to-intracranial; $\mathrm{PComA}=$ posterior communicating artery; $\mathrm{AComA}=$ anterior communicating artery; $\mathrm{CCF}=$ carotid-cavernous fistula; $\mathrm{MCA}$ = middle cerebral artery; ECA = external carotid artery; ICG-VA = Indocyanine green video angiography; $\mathrm{VA}=$ vertebral artery; PICA = posterior inferior cerebellar artery; $\mathrm{AVM}=$ arteriovenous malformation; $\mathrm{ACA}=$ anterior cerebral artery; $\mathrm{AChA}=$ anterior choroidal artery; $\mathrm{BA}=$ basilar artery; $\mathrm{LSO}=$ lateral supraorbital; AICA = anterior inferior cerebellar artery; STA = superficial temporal artery; SCA = superior cerebellar artery; PCA = posterior cerebral artery; TCD = transcranial doppler. 


\begin{tabular}{|c|c|c|c|}
\hline No. & Pathology & Operation & $\begin{array}{l}\text { Complexity of aneurysms, intraoperative strategy, and } \\
\text { Flow } 800 \text { findings }\end{array}$ \\
\hline 30 & $\begin{array}{l}\text { Left ICA C6 } \\
\text { aneurysm }\end{array}$ & Clipping & $\begin{array}{l}\text { We grinded the bone carefully to expose the aneurysm, } \\
\text { which was located inside of the anterior clinoid process. } \\
\text { We temporarily blocked the ICA and clipped the aneurysm } \\
\text { with a cross-window right-angle clip. Intraoperative FLOW } \\
800 \text { analysis confirmed the clipping of the aneurysm was } \\
\text { satisfactory and the parent artery was unobstructed. }\end{array}$ \\
\hline 31 & $\begin{array}{l}\text { BA ruptured } \\
\text { aneurysm }\end{array}$ & Clipping & $\begin{array}{l}\text { The aneurysm was clipped with a } 940 \text {-aneurysm clip. } \\
\text { FLOW } 800 \text { analysis showed the clipping of the aneurysm } \\
\text { was satisfactory and the BA and bilateral PCAs were } \\
\text { unobstructed. }\end{array}$ \\
\hline 32 & $\begin{array}{l}\text { Left AICA } \\
\text { ruptured } \\
\text { aneurysm }\end{array}$ & Clipping & $\begin{array}{l}\text { Under the microscope, the left VII, VIII nerves were } \\
\text { exposed, and we found the left AlCA went through the VII, } \\
\text { VIII nerves. The aneurysm was seen on the ventral side of } \\
\text { VIII nerve. We carefully separated the aneurysm and } \\
\text { clipped it with a } 740 \text {-aneurysm clip. The important } \\
\text { structures such as facial nerve, acoustic nerve, trigeminal } \\
\text { nerve, posterior cranial nerves, brainstem were and the } \\
\text { petrosal vein were well preserved. Intraoperative FLOW } \\
800 \text { analysis showed that the parent artery was } \\
\text { unobstructed and there was no stenosis. }\end{array}$ \\
\hline \multicolumn{4}{|c|}{$\begin{array}{l}\text { ICA = internal carotid artery; high flow EC-IC = extracranial-to-intracranial; PComA = posterior } \\
\text { communicating artery; } A C \text { ComA = anterior communicating artery; CCF = carotid-cavernous fistula; MCA } \\
\text { = middle cerebral artery; ECA = external carotid artery; ICG-VA = Indocyanine green video angiography; } \\
\text { VA = vertebral artery; PICA = posterior inferior cerebellar artery; AVM = arteriovenous malformation; } \\
\text { ACA = anterior cerebral artery; AChA = anterior choroidal artery; BA = basilar artery; } \mathrm{LSO}=\text { lateral } \\
\text { supraorbital; AICA = anterior inferior cerebellar artery; STA = superficial temporal artery; SCA = superior } \\
\text { cerebellar artery; PCA = posterior cerebral artery; } \mathrm{TCD}=\text { transcranial doppler. }\end{array}$} \\
\hline
\end{tabular}

\section{Case 1}

A 61-year-old female patient (No. 3 in Table 2) presented with pulsatile exophthalmos and headache. Imaging detected one giant aneurysm $\left(25^{\star} 20 \mathrm{~mm}\right)$ with carotid-cavernous fistula (CCF) located on the left ICA (Fig. 1A-D). The left pterional approach was used for craniotomy. After complete microsurgical dissection of the aneurysms, the patient underwent intraoperative ICG-VA and FLOW 800 analysis (Fig. 1E-G). The left forearm was cut straight along the radial artery (RA) which was dissociated, washed with papaverine, and soaked in normal saline for bypass. Then, we took a straight incision in the neck and exposed the left CCA upward to the bifurcations of ICA and ECA. Afterwards, Following high flow extracranial-to-intracranial (EC-IC) bypass (M2-RA-ICA), we trapped aneurysm at initial part of ICA and the distal end of the PComA. Intraoperative ICG-VA, FLOW 800 analysis were repeated to confirm the aneurysm disappeared and the bypass vascular was fluent (Fig. 1H-J). In addition, intraoperative ultrasound was applied to reconfirm the anastomotic graft was unobstructed. After operation, there was no hemorrhage in the operative area on CT. The complete obliteration of the aneurysm and the patency of bypass were confirmed by postoperative CTA two days after surgery. The cerebral blood perfusion was also good on CTP. At discharge, the patient had signs of injury to the left oculomotor nerve, but the symptoms of eyeball pulsation and headache had basically disappeared. 


\section{Case 2}

A 62-year-old male patient (No. 22 in Table 2 ) was admitted due to an aneurysm of about $4 * 3 \mathrm{~mm}$ in size found in the left posterior communicating artery (L-PComA). CTA and DSA indicated that the aneurysm was located outside of the left ICA, pointing outward and downward, with obvious peripheral adhesion. The PComA went out from the proximal aneurysm neck (Fig. 2A-C). The optic nerve, ICA, PComA and the lateral of aneurysm were exposed after frontal lobe was dragged by brain spatula. After the surrounding arachnoid was separated carefully, the proximal part of PComA and the body of the aneurysm are visible. We dissociated the aneurysm neck and clipped the aneurysm neck with a cross-window aneurysm clip (Fig. 2D-G). Intraoperative FLOW 800 analysis indicated that the clipping was satisfactory and the parent artery was unobstructed (Fig. $2 \mathrm{H}-\mathrm{I})$.

\section{Case 3}

During routine CT and CTA screen in a 62-year-old male (No. 26 in Table 2), a right MCA aneurysm which was located in the bifurcation of the M2 segment of the right MCA was detected (20*18 mm) (Fig. 3A-D). The right frontotemporal arc incision was used intraoperatively. The right M2 and M3 were exposed after separating the side crack. An aneurysm of about $2.0 * 1.8 \mathrm{~cm}$ in size at the $\mathrm{M} 2$ bifurcation can be clearly seen. Two M3 emanate from the aneurysm. Decided to dissociate the superficial temporal artery because aneurysm is hard to shape and low flow extracranial-to-intracranial (EC-IC) bypass (superficial temporal artery-M3) was adopted. During the surgery, microvascular Doppler exploration revealed obvious blood flow in M3 while high blood flow resistance was showed in M2. Aneurysms were temporarily isolated due to another M3 reflux condition available. Repeated ICG-VA and FLOW800 suggested unobstructed blood flow of bypass M3, however, the un-bypass M3 had collateral blood supply artery (Fig. 3E-G). After the M3 branch was blocked with aneurysm clip, the aneurysm was removed and ICG-VA was repeated. FLOW 800 suggested that the aneurysm disappeared and the bypass artery had good blood flow (Fig. 3H-K). No infarction and fresh bleeding were seen on postoperative CT scans. TCD re-examination on the first day after the operation showed that the blood flow of the bypass vessel was unobstructed. CTA showed fluent blood flow of the bypass artery three days later, and MR examination showed no obvious infarction eight days later. DSA showed clear bypass blood flow and clear imaging of each branch of the right middle cerebral artery ten days later. The patient showed no obvious positive symptoms at discharge.

\section{Discussion}

In the treament of complex intracranial aneurysms, clipping, bypass and resection are the main surgical methods. And there are more options for aneurysm clipping in the present clinical work. Microsurgical clipping of cerebral aneurysms is likely to lead to cerebral infarction. According to relevant reports, the average incidence of cerebral infarction after surgical clipping of unruptured aneurysms is $11.3 \%(10.9 \%$ $\left.(208 / 1917)^{[19]}, 12.1 \%(44 / 363)^{[20]}, 12.6 \%(43 / 338)^{[21]}\right)$, while the average incidence of cerebral infarction after surgical clipping of ruptured aneurysms is $37.9 \%\left(21.0 \%(33 / 157)^{[22]}, 21.0 \%(33 / 157)^{[23]}\right)$. It can be seen that the incidence of cerebral infarction after clipping ruptured aneurysms is 3 times as high as 
unruptured aneurysms, which is related to the imperfect preoperative preparation and the lack of effective intraoperative means to evaluate the hemodynamic changes after aneurysm clipping. Therefore, it is very important to find a way to instruct the surgeon to adjust the intraoperative aneurysm clip and change the surgical method.

ICG is a near infrared fluorescent tricarbonine dye used in medical diagnosis. The dye is injected intravenously, binds tightly to plasma proteins, and is confined to the endovascular system. ICG is eliminated from the bile circulation only by hepatic metabolism with a half-life of 3 to 4 minutes ${ }^{[24]}$. ICGVA can observe the patency of blood vessels, complete clipping of aneurysms and the blood flow in each branch of blood vessels during the operation. Combination with the high-resolution images of the microscope, it can provide basis for clinicians to timely adjust the surgical plan or aneurysm clip during the operation. Although these merits make ICG-VA look promising, the lack of measurable quantitative values of ICG-VAG raised much concern. It has been reported that the consistency rate between ICG-VA and the intraoperative DSA is only $75 \%\left[{ }^{[10]}\right.$. Which requires further development of auxiliary tools to help improve the accuracy of ICG-VA. Flow 800 is an excellent semi-quantitative analysis software. By analyzing the fluorescence intensity after ICG injection and generating color coded graphs, the direction of blood flow and the patency degree of blood vessels can be determined according to the intensity of the color, especially the effective evaluation of blood vessels at the surgical site and optimized assessment of the vascular anatomy. ${ }^{[11]}$. FLOW 800 technology does not measure the absolute rate of flow but rather provides reliable comparable flow amongst the vasculature within the field of view as mentioned. Although FLOW 800 has been reported in many literatures in the current surgical operation, the application of FLOW 800 in intracranial complex aneurysms has been rarely reported, especially for the lack of quantitative analysis of flow data during aneurysm surgery. DSA can evaluate the effect of the operation better, but due to its time-consuming and complex operation, the DSA scanning is obviously not realistic in the procedure, so that many hospitals have abandoned the gold standard. When combination of the information generated by ICG-VA and the FLOW800 generated blood flow perfusion, it can be used as a supplement to provide the blood perfusion of the small arteries, and the surgical procedure before the operation is performed by the surgeon to evaluate the effect of the operation in time, and even to remedy the adverse events of the operation. What is more important is that due to the safety and timeliness of ICG-VA, doctors can perform angiography several times during the operation to continuously improve the operation quality through FLOW 800. By repeating ICG-VA, the stenosis of the parent artery, branch artery and bypass artery vessels and the velocity of blood flow in the artery after the critical surgical procedure can be determined. In the long run, in combination with the patients with the postoperative CT scan, TCD, DSA and MR scan, strong guidance can be provided for the treatment rehabilitation training of patients.

However, due to too much subjectivity during the intraoperative application of ICG-VA and FLOW 800, Such as blood clots or brain tissue blocking arteries, or arteries are too deep to be observed, which requires strong intraoperative decision-making ability of the surgeon. Meanwhile, there are also problems caused by unhealthy arteries of patients, such as calcification, atherosclerosis, and abnormal thickening 
of blood vessel walls, resulting in the observed weakening of fluorescence intensity, which also presents a new challenge to the intraoperative judgment of surgeon. It can be seen that although FLOW 800 is a good supplement to ICG-VA, there are still many imperfections to be improved. In brief, although FLOW 800 analysis can provide additional information for ICG-VA to detect hemodynamically related arterial stenosis during surgery, it may further improve the safety of microsurgical clipping. However, since there are few clinical cases available, larger comparative studies are necessary to provide clear conclusions about this approach.

\section{Conclusion}

ICG-VA and FLOW 800 provide high resolution images and it produce the direction and relative magnitude of flow to and from vascular lesions for surgeon. Which can be used to evaluate the patency of the parent artery, branch artery and bypass artery to change the decision during surgery. However, the information is subjective and needs to be judged by surgeons with rich experience. Therefore, the beneficial effects of FLOW800 and aneurysm patient recovery need to be further demonstrated.

\section{Abbreviations}

AcomA = anterior communicating artery;

$\mathrm{ACA}=$ anterior cerebral artery;

$\mathrm{AChA}=$ anterior choroidal artery;

AICA = anterior inferior cerebellar artery;

$\mathrm{AVM}=$ arteriovenous malformation;

$\mathrm{BA}=$ basilar artery;

CCF = carotid-cavernous fistula;

$\mathrm{CT}$ = computed tomography;

DSA = digital subtraction angiography;

$\mathrm{ECA}=$ external carotid artery;

EC-IC = extracranial-to-intracranial;

GCS = Glasgow Coma Scale;

ICA = internal cerebral artery;

ICG = indocyanine green; 


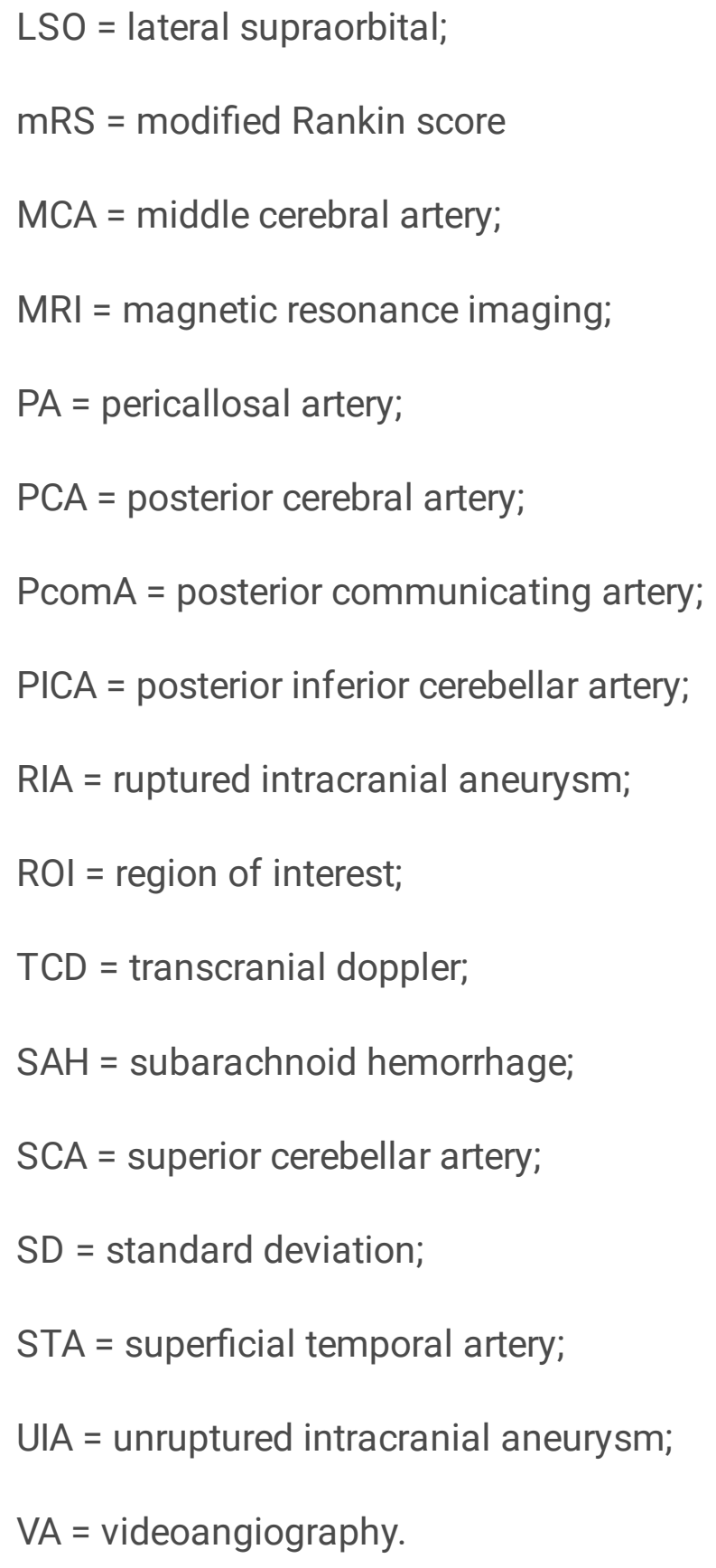

\section{Declarations}

\section{Ethics approval and consent to participate}

All procedures performed in studies involving human participants were in accordance with the ethical standards of the institutional and/or national research committee and with the 1964 Helsinki Declaration and its later amendments or comparable ethical standards. The study was approved by the Institutional Review Board of the First Affiliated Hospital of Soochow University. As this article is a retrospective study, ethics committees have been granted exemption of patient informed consent.

\section{Consent for publication}

Not applicable. 
Availability of data and materials

Please contact author for data requests.

\section{Competing interests}

The authors declare that they have no competing interests.

\section{Funding}

This work was supported by the Suzhou Health Talents Training Project (GSWS2019002).

\section{Authors' contributions}

ZW and ZQC was the principal investigator. TX and RMD designed the study and developed the analysis plan. BXG and ZLW contributed in writing of the article. WCY, CM and YZ revised the manuscript and polish the language.

\section{Acknowledgements}

Not applicable.

\section{Author's information}

${ }^{1}$ Department of Neurosurgery \& Brain and Nerve Research Laboratory, The First Affiliated Hospital of Soochow University, Suzhou, Jiangsu Province, 215006, China

${ }^{2}$ Department of Neurosurgery, Bozhou People's Hospital, Anhui Province, China

\section{References}

1. Hanel RA, Spetzler RF. Surgical treatment of complex intracranial aneurysms [J]. Neurosurgery. 2008;62(6 Suppl 3):1289-97. discussion 97 - 9.

2. Bracard S, Derelle AL, Tonnelet R, Barbier $C$, Proust $F$, Anxionnat R. [Endovascular treatment of giant intracranial aneurysms] [J]. Neurochirurgie. 2016;62(1):25-9.

3. Lejeune JP, Thines L, Proust F, Riegel B, Koussa M, Decoene C. Selective microsurgical treatment of giant intracranial aneurysms [J]. Neurochirurgie. 2016;62(1):30-7.

4. Lawton MT, Spetzler RF. Surgical strategies for giant intracranial aneurysms [J]. Acta neurochirurgica Supplement. 1999;72:141-56.

5. Raabe A, Beck J, Gerlach R, Zimmermann M, Seifert V. Near-infrared indocyanine green video angiography: a new method for intraoperative assessment of vascular flow [J]. Neurosurgery. 2003;52(1):132-9. discussion 9. 
6. Woitzik J, Horn P, Vajkoczy P, Schmiedek P. Intraoperative control of extracranial-intracranial bypass patency by near-infrared indocyanine green videoangiography [J]. Journal of neurosurgery. 2005;102(4):692-8.

7. Raabe A, Nakaji P, Beck J, Kim LJ, Hsu FP, Kamerman JD, et al. Prospective evaluation of surgical microscope-integrated intraoperative near-infrared indocyanine green videoangiography during aneurysm surgery [J]. Journal of neurosurgery. 2005;103(6):982-9.

8. Woitzik J, Pena-Tapia PG, Schneider UC, Vajkoczy P, Thome C. Cortical perfusion measurement by indocyanine-green videoangiography in patients undergoing hemicraniectomy for malignant stroke [J]. Stroke. 2006;37(6):1549-51.

9. Czabanka M, Pena-Tapia P, Schubert GA, Woitzik J, Vajkoczy P, Schmiedek P. Characterization of cortical microvascularization in adult moyamoya disease [J]. Stroke. 2008;39(6):1703-9.

10. Washington CW, Zipfel GJ, Chicoine MR, Derdeyn CP, Rich KM, Moran CJ, et al. Comparing indocyanine green videoangiography to the gold standard of intraoperative digital subtraction angiography used in aneurysm surgery [J]. Journal of neurosurgery. 2013;118(2):420-7.

11. Shah KJ, Cohen-Gadol AA. The Application of FLOW 800 ICG Videoangiography Color Maps for Neurovascular Surgery and Intraoperative Decision Making [J]. World neurosurgery. 2019;122:e186e97.

12. Fukuda K, Kataoka H, Nakajima N, Masuoka J, Satow T, lihara K. Efficacy of FLOW 800 with indocyanine green videoangiography for the quantitative assessment of flow dynamics in cerebral arteriovenous malformation surgery [J]. World neurosurgery. 2015;83(2):203-10.

13. Kalyvas J, Spetzler RF. Does FLOW 800 technology improve the utility of indocyanine green videoangiography in cerebral arteriovenous malformation surgery? [J]. World neurosurgery. 2015;83(2):147-8.

14. Goertz L, Hof M, Timmer M, Schulte AP, Kabbasch C, Krischek B, et al. Application of Intraoperative FLOW 800 Indocyanine Green Videoangiography Color-Coded Maps for Microsurgical Clipping of Intracranial Aneurysms [J]. World neurosurgery. 2019;131:e192-200.

15. Ye X, Liu XJ, Ma L, Liu LT, Wang WL, Wang S, et al. Clinical values of intraoperative indocyanine green fluorescence video angiography with Flow 800 software in cerebrovascular surgery [J]. Chin Med J. 2013;126(22):4232-7.

16. Rennert RC, Strickland BA, Ravina K, Bakhsheshian J, Fredrickson V, Carey J, et al. Intraoperative Assessment of Cortical Perfusion After Intracranial-To-Intracranial and Extracranial-To-Intracranial Bypass for Complex Cerebral Aneurysms Using Flow 800 [J]. Operative neurosurgery. 2019;16(5):583-92.

17. Viski S, Olah L. Use of Transcranial Doppler in Intensive Care Unit [J]. Journal of critical care medicine. 2017;3(3):99-104.

18. Raabe A, Beck J, Seifert V. Technique and image quality of intraoperative indocyanine green angiography during aneurysm surgery using surgical microscope integrated near-infrared video technology [J]. Zentralbl Neurochir. 2005;66(1):1-6. discussion 7-8. 
19. Wiebers DO, Whisnant JP, Huston J 3rd, Meissner I, Brown RD Jr, Piepgras DG, et al. Unruptured intracranial aneurysms: natural history, clinical outcome, and risks of surgical and endovascular treatment [J]. Lancet. 2003;362(9378):103-10.

20. Kunz M, Bakhshai Y, Zausinger S, Fesl G, Janssen H, Bruckmann H, et al. Interdisciplinary treatment of unruptured intracranial aneurysms: impact of intraprocedural rupture and ischemia in 563 aneurysms [J]. Journal of neurology. 2013;260(5):1304-13.

21. Li M, Wu J, Chen X, Jiang P, Yang F, Ma Y, et al. Symptomatic and silent cerebral infarction following surgical clipping of unruptured intracranial aneurysms: incidence, risk factors, and clinical outcome [J]. Neurosurgical review. 2018;41(2):675-82.

22. Goertz L, Kabbasch C, Borggrefe J, Hamisch C, Telentschak S, von Spreckelsen N, et al. Preoperative Three-Dimensional Angiography May Reduce Ischemic Complications During Clipping of Ruptured Intracranial Aneurysms [J]. World neurosurgery. 2018;120:e1163-e70.

23. Yao PS, Chen GR, Zheng SF, Kang DZ. Predictors of Postoperative Cerebral Ischemia in Patients with Ruptured Anterior Communicating Artery Aneurysms [J]. World neurosurgery. 2017;103:241-7.

24. Oda J, Kato Y, Chen SF, Sodhiya P, Watabe T, Imizu S, et al. Intraoperative near-infrared indocyanine green-videoangiography (ICG-VA) and graphic analysis of fluorescence intensity in cerebral aneurysm surgery [J]. Journal of clinical neuroscience: official journal of the Neurosurgical Society of Australasia. 2011;18(8):1097-100.

\section{Figures}




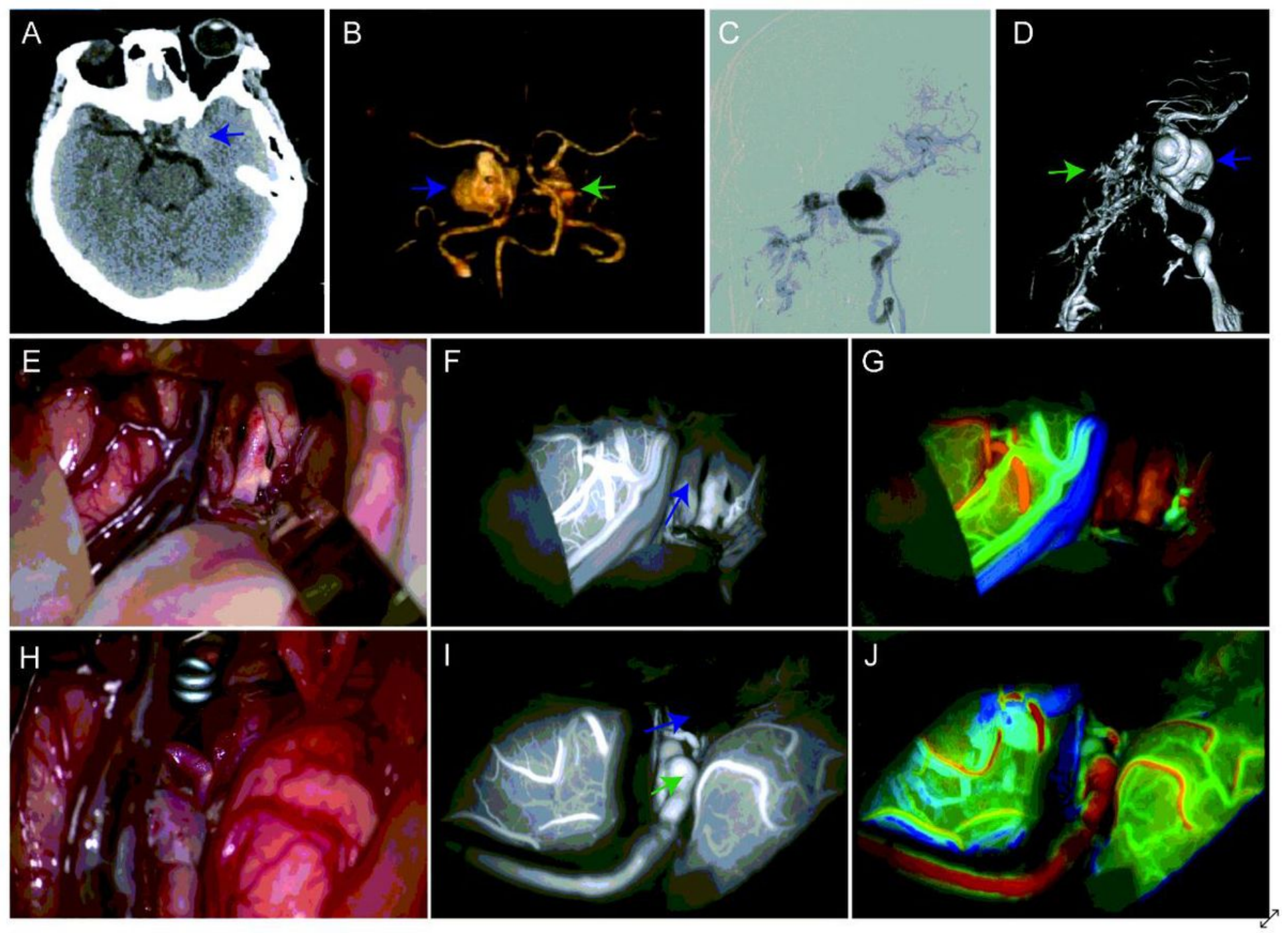

Figure 1

A left internal carotid artery (ICA) C4 segment unruptured giant aneurysm $\left(25^{\star} 20 \mathrm{~mm}\right.$, blue arrow) with carotid-cavernous fistula (CCF, green arrow) was diagnosed by computed tomography (A), computed tomography angiography (B), digital subtraction angiography (C), and 3-dimensional rotational angiography (D). During surgery, the aneurysm was exposed (E). Then, conventional indocyanine green video angiography (ICG-VA) showed aneurysm (F, blue arrow), parent artery and peripheral blood vessels. FLOW 800 provided a color-coded map that displayed the brain vessels in contrasting colors depending on the fluorescence appearance after ICG injection (arteries: red, cortical capillaries: yellow/green, veins: blue) (G). After high flow extracranial-to-intracranial (EC-IC) bypass (middle cerebral artery M3 segmentradial artery-ICA), we isolated aneurysm at ICA initial part and ICA before posterior communicating artery (PComA) branching $(\mathrm{H})$. Another ICG-VA $(\mathrm{I})$ and FLOW 800 analysis $(\mathrm{J})$ showed the aneurysm disappeared (blue arrow) and the bypass vessel was fluent (green arrow). 

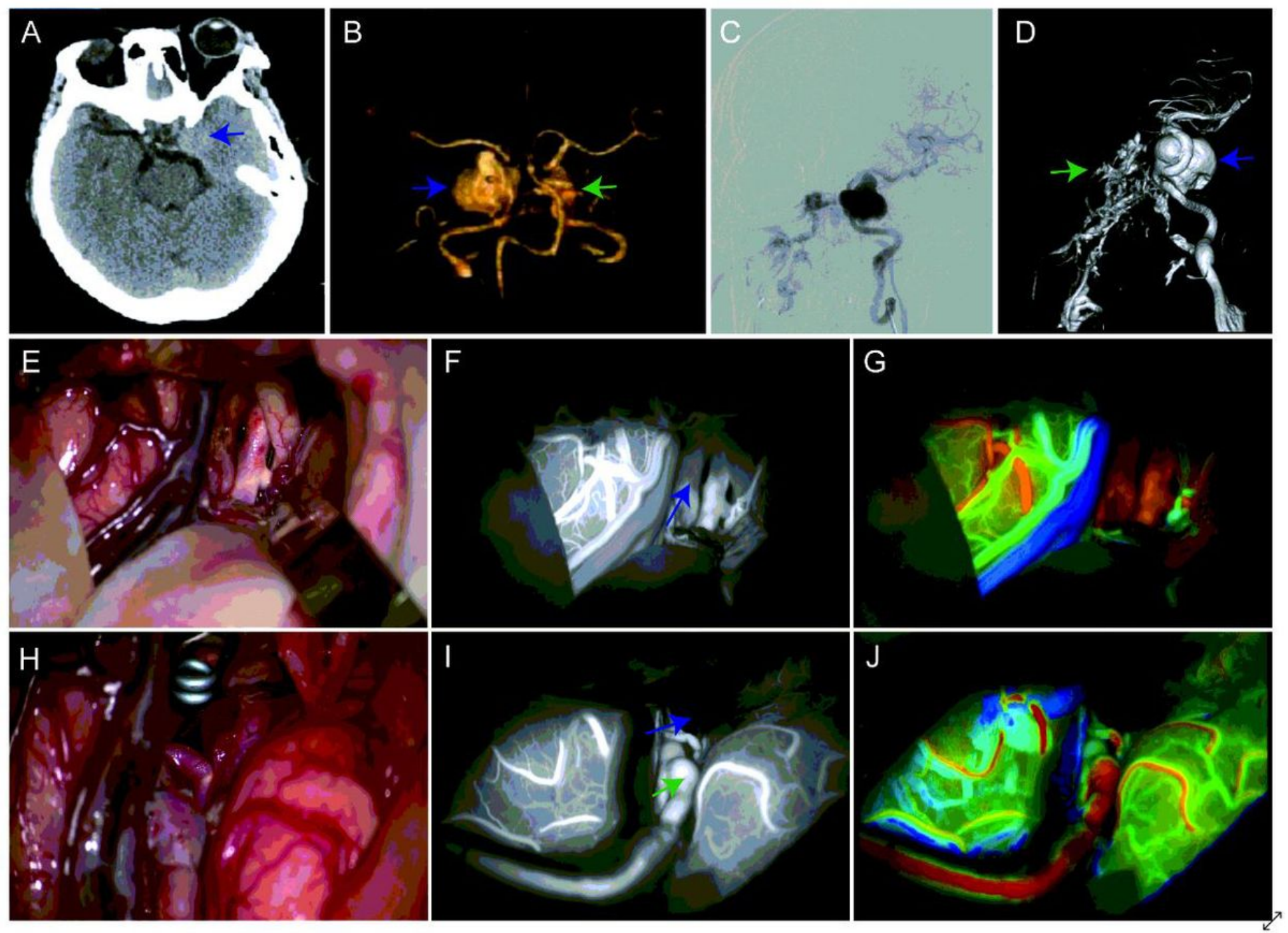

Figure 1

A left internal carotid artery (ICA) C4 segment unruptured giant aneurysm $\left(25^{\star} 20 \mathrm{~mm}\right.$, blue arrow) with carotid-cavernous fistula (CCF, green arrow) was diagnosed by computed tomography (A), computed tomography angiography (B), digital subtraction angiography (C), and 3-dimensional rotational angiography (D). During surgery, the aneurysm was exposed (E). Then, conventional indocyanine green video angiography (ICG-VA) showed aneurysm (F, blue arrow), parent artery and peripheral blood vessels. FLOW 800 provided a color-coded map that displayed the brain vessels in contrasting colors depending on the fluorescence appearance after ICG injection (arteries: red, cortical capillaries: yellow/green, veins: blue) (G). After high flow extracranial-to-intracranial (EC-IC) bypass (middle cerebral artery M3 segmentradial artery-ICA), we isolated aneurysm at ICA initial part and ICA before posterior communicating artery (PComA) branching $(\mathrm{H})$. Another ICG-VA (I) and FLOW 800 analysis $(\mathrm{J})$ showed the aneurysm disappeared (blue arrow) and the bypass vessel was fluent (green arrow). 


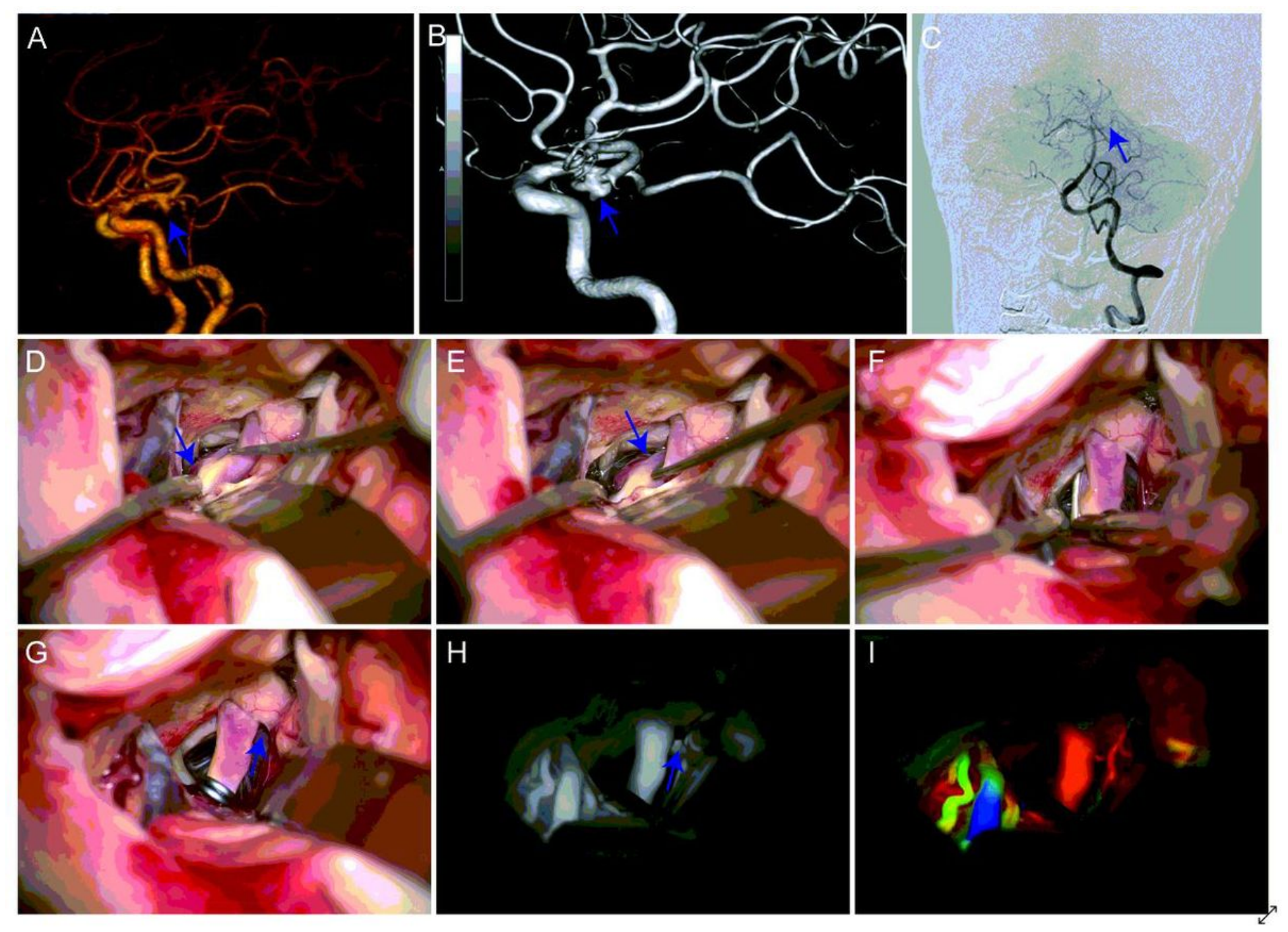

\section{Figure 2}

A left posterior communicating artery (PComA) aneurysm ( $4 * 3 \mathrm{~mm}$, blue arrow) was diagnosed by computed tomography angiography (A), digital subtraction angiography (B) During surgery, the lateral (D) and body (E) of aneurysm was exposed. The aneurysm neck was clipped with a cross-window aneurysm clip (F) and PComA was visible (G) (blue arrow). Conventional ICG-VA (H) and FLOW 800 analysis (I) showed clipping was satisfactory and the parent artery was unobstructed (blue arrow). 


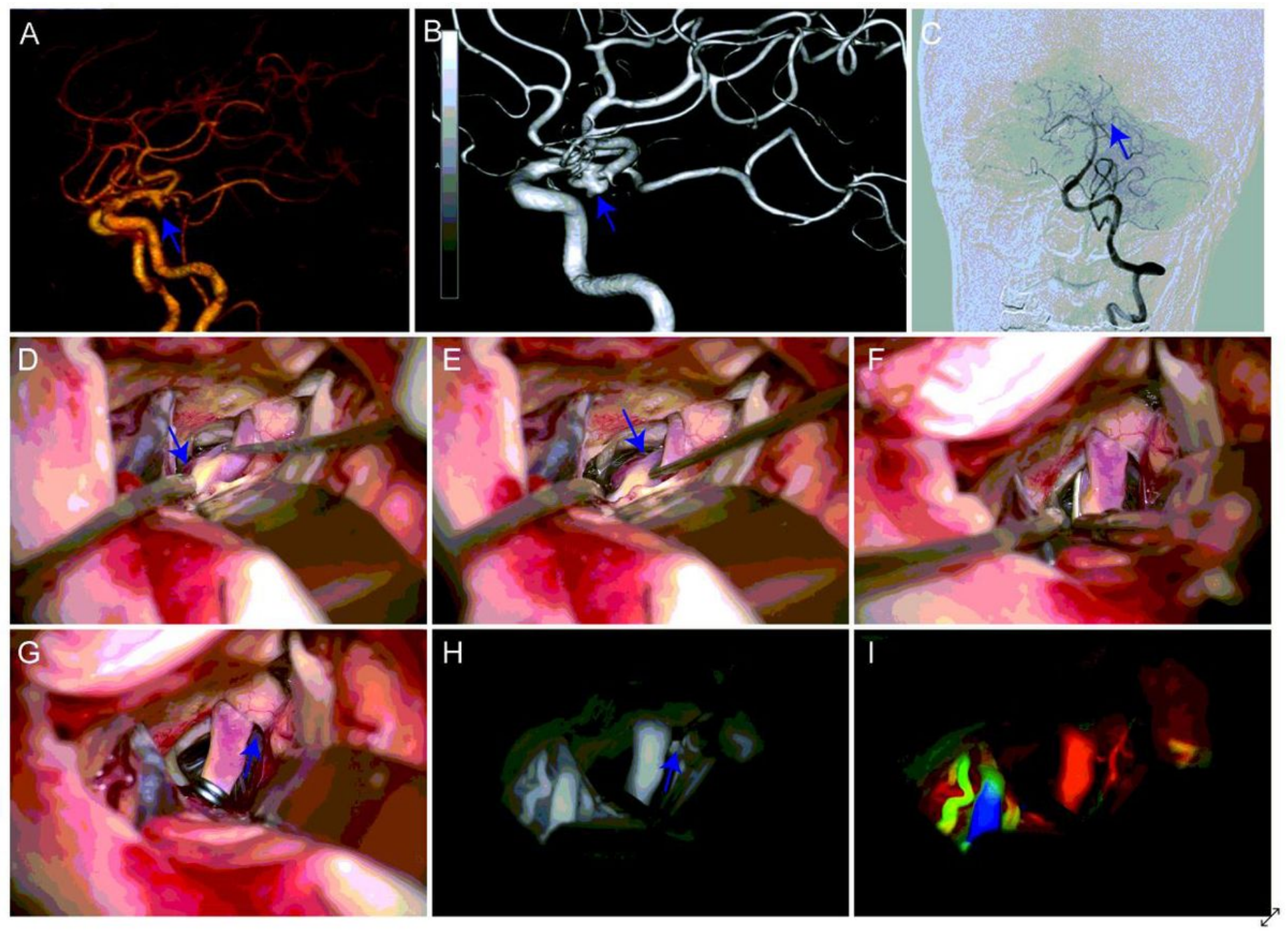

Figure 2

A left posterior communicating artery (PComA) aneurysm ( $4 * 3 \mathrm{~mm}$, blue arrow) was diagnosed by computed tomography angiography (A), digital subtraction angiography (B) During surgery, the lateral (D) and body (E) of aneurysm was exposed. The aneurysm neck was clipped with a cross-window aneurysm clip (F) and PComA was visible (G) (blue arrow). Conventional ICG-VA (H) and FLOW 800 analysis (I) showed clipping was satisfactory and the parent artery was unobstructed (blue arrow). 

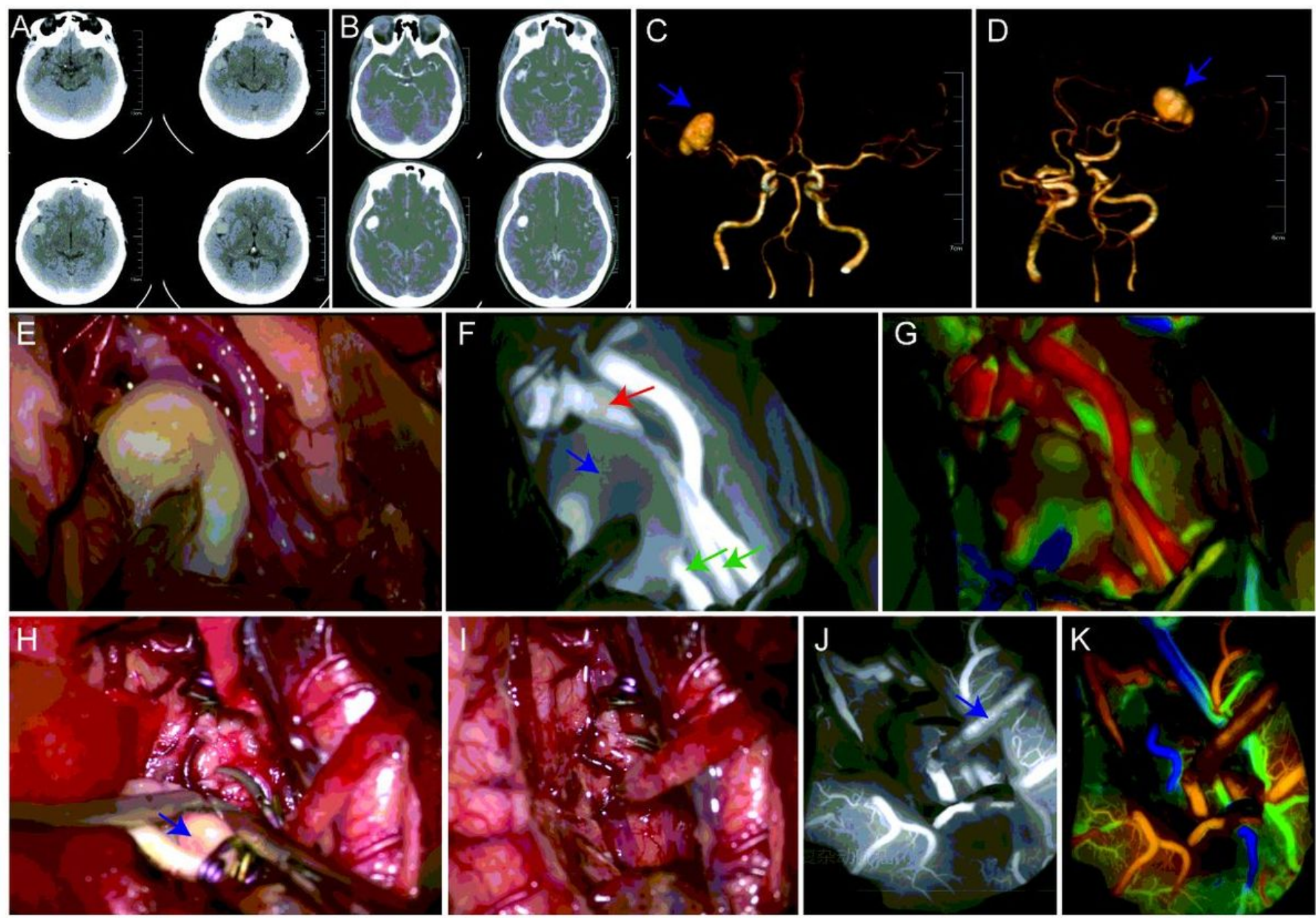

\section{Figure 3}

A Right Middle cerebral artery (MCA) M2 bifurcation unruptured large aneurysm (20*18 mm, blue arrow) with two M3 branches starting from the aneurysm was diagnosed by computed tomography ( $A$ and $B$ ) and computed tomography angiography $(C$ and $D)$. During surgery, we exposed the aneurysm and performed electrocoagulation to shrink it $(\mathrm{E})$. Then, conventional indocyanine green video angiography (ICG-VA) showed aneurysm (F, blue arrow), parent artery including M2 (red arrow) and two M3 (green arrows). FLOW 800 provided a color-coded map that displayed the brain vessels in contrasting colors depending on the fluorescence appearance after ICG injection (arteries: red, cortical capillaries: yellow/green, veins: blue) (G). After low flow extracranial-to-intracranial (EC-IC) bypass (superficial temporal artery-M3), we isolated and resected the aneurysm ( $\mathrm{H}$ and I, blue arrow). Another ICG-VA $(\mathrm{J})$ and FLOW 800 analysis $(K)$ showed the aneurysm disappeared and the bypass vessel was unobstructed (blue arrow). 

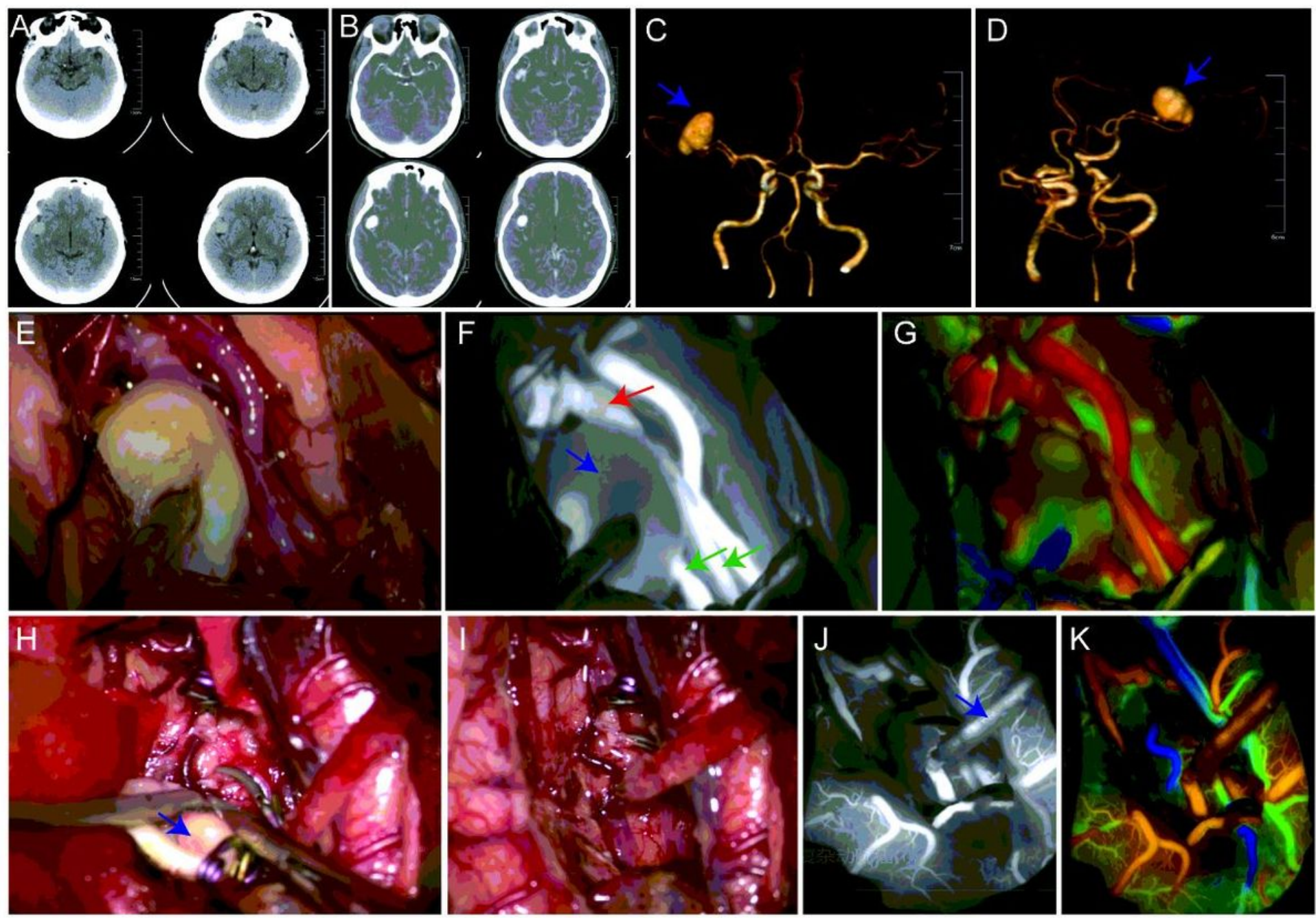

\section{Figure 3}

A Right Middle cerebral artery (MCA) M2 bifurcation unruptured large aneurysm (20*18 mm, blue arrow) with two M3 branches starting from the aneurysm was diagnosed by computed tomography ( $A$ and $B$ ) and computed tomography angiography $(C$ and $D)$. During surgery, we exposed the aneurysm and performed electrocoagulation to shrink it $(\mathrm{E})$. Then, conventional indocyanine green video angiography (ICG-VA) showed aneurysm (F, blue arrow), parent artery including M2 (red arrow) and two M3 (green arrows). FLOW 800 provided a color-coded map that displayed the brain vessels in contrasting colors depending on the fluorescence appearance after ICG injection (arteries: red, cortical capillaries: yellow/green, veins: blue) (G). After low flow extracranial-to-intracranial (EC-IC) bypass (superficial temporal artery-M3), we isolated and resected the aneurysm ( $\mathrm{H}$ and I, blue arrow). Another ICG-VA $(\mathrm{J})$ and FLOW 800 analysis $(K)$ showed the aneurysm disappeared and the bypass vessel was unobstructed (blue arrow). 